\section{Dust-up in Nevada}

$\mathrm{IT}_{\mathrm{T}}$ is rare for a scientific article to elicit ferocious response within wecks of publication-most experimental studies and theorics are poised on a superstructure of unique apparatus or theoretical formalism not immediately accessible to the reader. The experience of Emiliani, Harrison and Swanson of the University of Miami is thus all the more singular. In Science $(165,1255 ; 1969)$ they postulated that underground nuclear test detonations in Nevada were causing increased earthquake activity at distances out to $860 \mathrm{~km}$ from the test site. They also suggested that such explosions might be useful in controlling earthquake activity.

Predictably they have received a sharp rap over the knuckles, and three rejoinders (Science, 16\%, 1011; 1970) have been published signed by no fewer than nine luminaries of the seismological world. The whole seismological staff of the California Institute of Technology add their weighty names to one such letter. The experience is perhaps more of a psychological than a geophysical happening-it reflects things felt rather than things known, and it reveals the danger of attempting to copyright what is no more than coffeeroom musing.

The question of destressing a region by some explosive process arose in 1963, when it was appreciated that some explosions have an earthquake type of radiation pattern. Recent large nuclear tests in. Nevada have triggered near-in (less than $20 \mathrm{~km}$ away) earthquakes by the thousand, a phenomenon which is universally known but little understood. Do the earthquakes release pre-existing stress or only stress accumulations from the explosion itself? Nobody knows for sure and a coordinated programme of earthquake control, such as the United States hovers on the brink of supporting, must regard this as one of its priorities. Hence, probably, a certain amount of irritation that Emiliani et al. should claim originality for their suggestion of earthquake control. To their credit they have made a full retraction of their claim to be the first to suggest it.

The issue of increased seismicity is more thorny, and this is not the first time that feathers have flown. For the past fifty years people have hunted for periodicities in earthquakes as a step towards their prediction. Such claims for periodicities have tended to diminish with the advent of improved facilities for harmonic analysis, but some apparent periodicities remain with us and call for continued study. One prerequisite, insufficiently realized, is the need to find a scoring system so that events of different magnitudes are given appropriate weight. Straightforward counting is not enough, and may be highly dependent on the network of stations used.

Another decision that needs to be taken concerns aftershocks of large earthquakes. Do they score in the same way as main shocks? Is the frequency of aftershocks controlled only by the nature of the main shock. or could a large shock render an area particularly prone to any periodic forces such as Earth tides? These questions, posed many times in periodicity studies, apply with equal force to the arguments of Emiliani et al. Credit where credit is due, the authors respond to their crities by showing data called for and on the basis of this there is a statistically significant increase in activity out to $800 \mathrm{~km}$ in the first 32 hours after a test. But there is no indication of magnitude of event, size of relevant explosions nor an adequate analysis of the incidence of aftershocks. The implications of what Emiliani et al. have said are great, but very few would yet attach any label other than "not proven" to the idea. A more sophisticated approach than straight counting is needed.

\section{PLANETARY NEBULAE Doublets give Electron Density}

by our Astronomy Correspondent

A METHOD of determining electron densities in planetary nebulae by observing the ratios of certain line intensities has been described by H. E. Saraph and M. J. Seaton, and an account of the work was given by Professor Seaton at last week's meeting of the Royal Astronomical Society. The technique is to measure the intensities of the two lines in certain doublets. and it seems that although the intensity ratio is insensitive to temperature, the ratio is very sensitive to electron density. The case of the OII doublet-3726 $\AA$ and $3729 \AA$--has been studied for several years, but Saraph and Seaton have extended the electron density measurements to other doublets which have not been studied in detail before.

One advantage of their new set of doublets is that the separations are greater than for the OII lines so that spectrograph dispersions need not be so high as for the OII case. The larger separation also means that electron density measurements can sometimes be taken in galaxies where the OII doublet cannot be resolved because of Doppler broadening.

The doublets which Saraph and Seaton discuss are SII (6731 and $6717 \AA$ ), CIIII (5538 and $5518 \AA$ ), ArIV (4740 and $4711 \AA$ ), and $K V(4163$ and $4123 \AA$ ). With the exception of SII, the intensity ratio for each doublet seems to have a larger sensitivity to elcetron densitythan OII, for a wide range of densities at least, and in particular the sensitivities are greatest around the electron density values which are typical of many planetary nebulae.

Actual measurements reported by Saraph and Seaton show good agrcement between electron densities obtained from different ions. Observations of IC 418. for example, give values of $\log n_{\mathrm{e}}$ of $3 \cdot 9, \leq 4 \cdot 4$ and $4 \cdot 1$ from the SII, OII and CIIII doublets respectively. In objects of high excitation the situation seems to be 